\title{
The Efficacy of Non-Vitamin K Antagonist Oral Anticoagulants in the Prevention of Left Atrial Thrombus in Patients With Atrial Fibrillation Compared With Vitamin K Antagonists: A Meta-Analysis
}

\author{
Jun Liu, MD,${ }^{1}$ Yi-Ping Wu, MD,${ }^{2}$ Shu-Rui Li, MD,${ }^{1}$ Li-Hong Song, MD, ${ }^{3}$ Cheng-Dong Hu, $\mathrm{MD}^{4}$
}

${ }^{1}$ Department of Geratology, Handan Central Hospital, Hebei, Handan, China; ${ }^{2}$ Department of Neurology, Handan Central Hospital, Hebei, Handan, China; ${ }^{3}$ Imaging Department, Handan Central Hospital, Hebei, Handan, China; ${ }^{4}$ Department of Orthopedics, Handan Central Hospital, Hebei, Handan China

\section{ABSTRACT}

Background: There is still a paucity of data on the efficacy of non-vitamin $\mathrm{K}$ antagonist oral anticoagulants (NOACs) in the prevention of left atrial thrombus (LAT) formation before cardioversion or catheter ablation. To assess the efficacy of NOACs in the prevention of LAT in patients with nonvalvular atrial fibrillation (NVAF) compared with vitamin $\mathrm{K}$ antagonists (VKAs), we conducted a meta-analysis.

Methods: We searched PubMed, Embase, and the Cochrane Library databases. For meta-analysis, dichotomous variables were analyzed by using the odds ratios (OR) computed using the Mantel Haenszel method (random models). All results were reported with 95\% confidence intervals (CI).

Results: A total of 13 studies (one randomized controlled investigation and 12 observational studies) were included in the meta-analysis. There was no statistically significant difference between the NOACs and VKAs groups with respect to the odds of LAT/LAAT formations (OR 0.79; 95\% CI: $0.52-1.21 ; P=.29 ;\left(\mathrm{I}^{2}=14 \%\right)$.

Conclusions: NOACs were as effective as VKAs in the prevention of LAT/LAAT formation in patients with NVAF. Though patients on NOACs therapy showed a lower incidence of LAT/LAAT formation compared with VKAs, it was not significant $(P=.29)$.

\section{INTRODUCTION}

Atrial fibrillation (AF) is one of the most common cardiac arrhythmias. Hypertension, aging, and congestive heart failure are all strong clinical predictors of AF [Schotten 2011]. Atrial contractile dysfunction in AF patients contributes to the formation of left atrial thrombus. One study suggests that LAT is present in $10 \%$ of patients with AF, causing a 3.5 -fold increased risk of stroke and systemic embolism [Di Minno 2016].

Anticoagulation therapy is an effective measure to prevent thromboembolism in patients with AF. According to guidelines,

Received fuly 19, 2020; accepted August 17, 2020.

Correspondence: Yi-Ping Wu, director, Department of Neurology, Handan Central Hospital, Hebei, Handan, China 056001 (e-mail:Wuyphd@163.com).

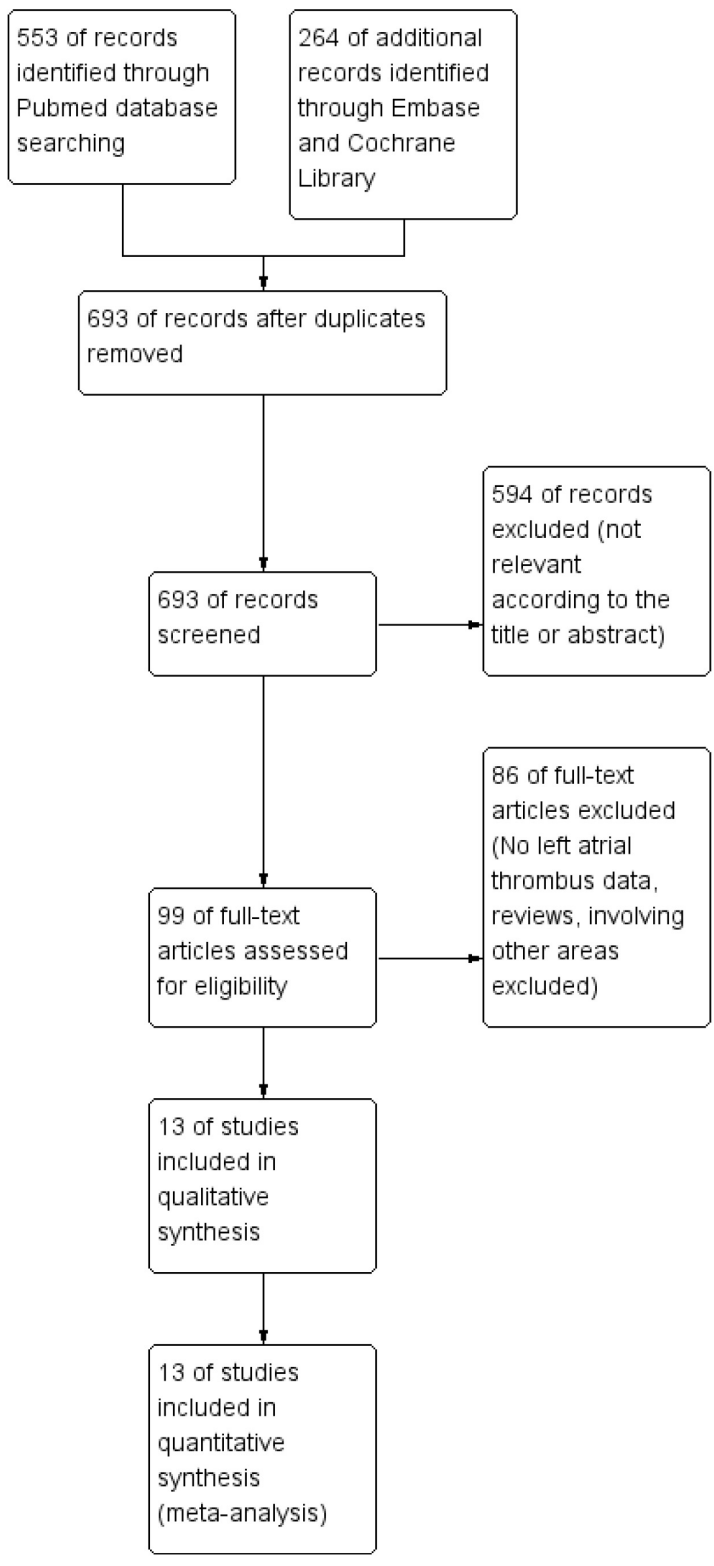

Figure 1. Presents the flowchart of search and the selection. 
Table 1. Baseline characteristics of the selected studies

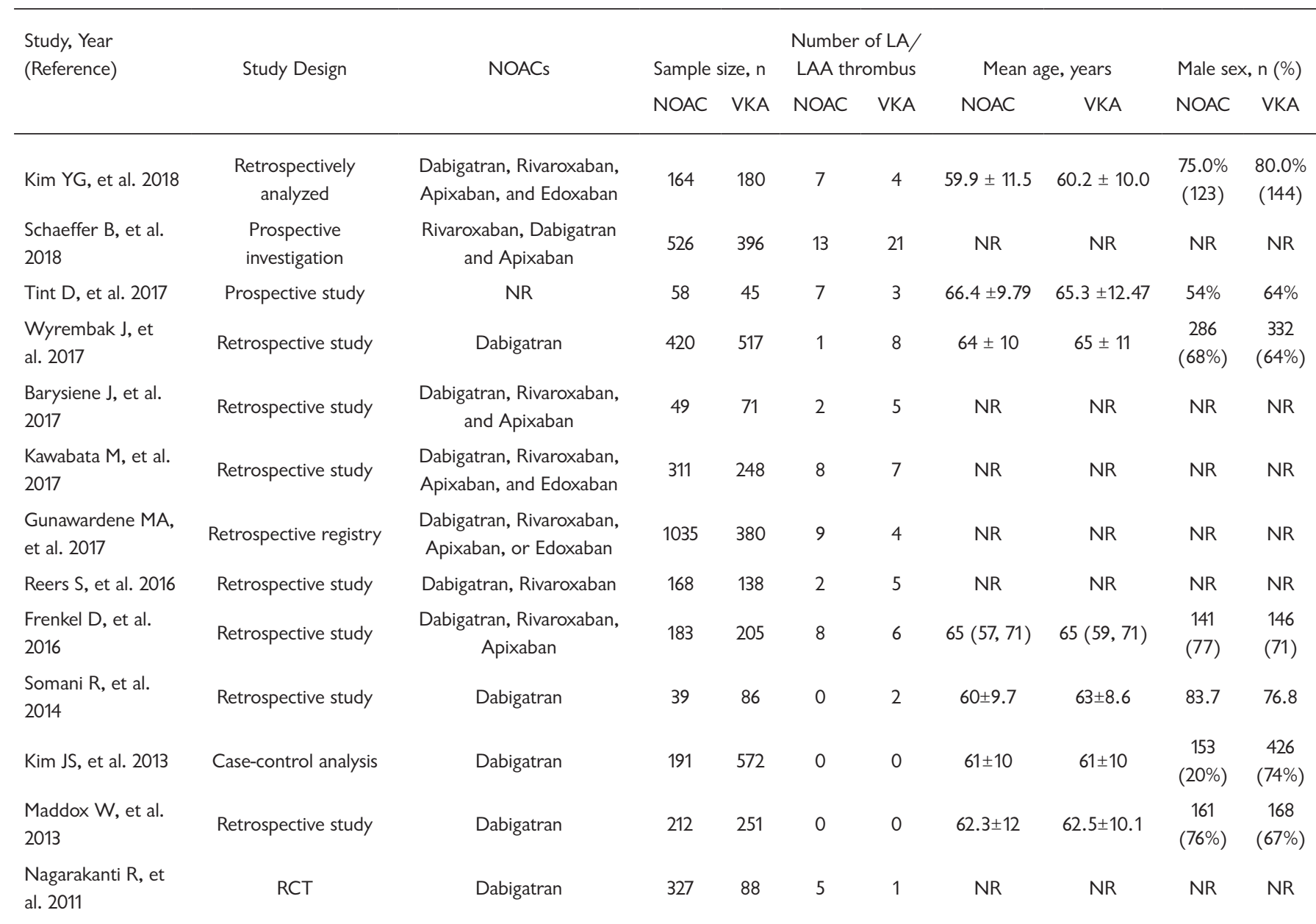

patients should be treated with either NOAC or VKA for at least three weeks before cardioversion [January 2019]. VKAs are traditional anticoagulant agents, among which, warfarin is the most widely used. However, it should be noted that warfarin is known to interact with food and many drugs, increasing the risk of adverse events. Inadequate monitoring of the international normalized ratio (INR) may lead to major bleeding and thrombosis recurrence. Furthermore, despite full anticoagulation on warfarin, 3.6\% of patients undergoing $\mathrm{AF}$ ablation had left atrial appendage (LAA) thrombus [Wallace 2010]. Non-vitamin K antagonist oral anticoagulants (NOACs) include a direct thrombin inhibitor (dabigatran) and factor Xa inhibitors (apixaban, rivaroxaban, and edoxaban). NOACs are more convenient than warfarin. They are promising alternatives to warfarin for prevention of stroke in non-valvular atrial fibrillation (NVAF) patients.

However, there's little data about left atrial thrombus detected in AF patients treated with NOACs. Research shows that the incidence of LAA thrombus in patients treated with NOACs is not negligible [Bertaglia 2017]. There is still controversy regarding the efficacy of NOACs for LAT prevention in NVAF compared with VKAs. Some studies indicate that the incidence of left atrial thrombus was lower in patients treated with NOAC than patients treated with warfarin [Yang 2019; Schaeffer 2018], while others do not [Reers 2016; Kawabata 2017]. Furthermore, the clinical trials of NOACs inconsistently reported race/ethnicity [Jackson 2018]. Therefore, we aimed to explore the efficacy of NOACs for LAT prevention in NVAF compared with VKAs.

\section{METHODS}

This meta-analysis was performed in accordance with Cochrane's Handbook guidelines [Higgins 2011], and the Preferred Reporting Items for Systematic Reviews and Meta-Analyses (PRISMA) Statement [Moher 2009].

Literature search: To identify eligible studies, we searched PubMed, Embase, and the Cochrane Library databases. MeSH terms included "atrial fibrillation," "anticoagulants," and "transesophageal echocardiography." The final literature search was performed on October 1, 2019.

Study selection inclusion and exclusion criteria: The inclusion criteria for our studies were as follows: (1) human studies; (2) patients with NVAF who were treated with NOACs 


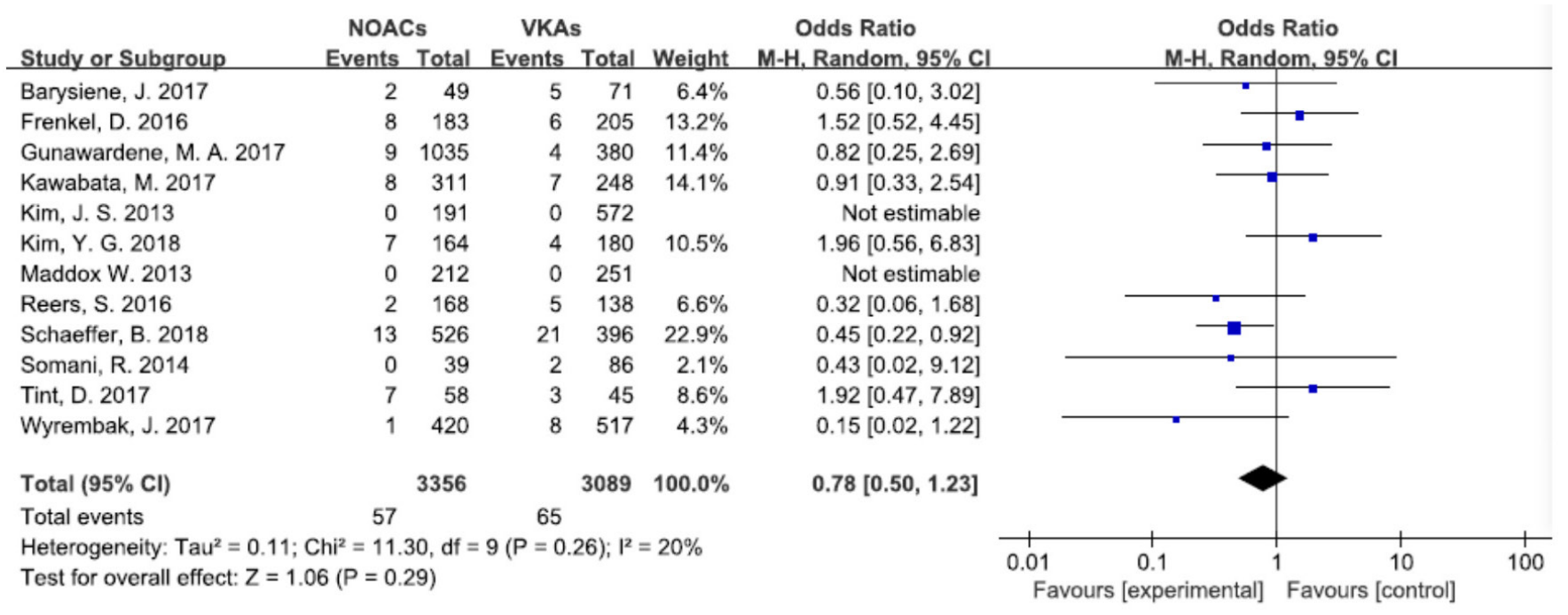

Figure 2. Forest plot of 12 observational studies for the meta-analysis of the frequencies of atrial thrombus between NOACs (experimental) and VKAs (control). There was no significant difference between the two groups with respect to the formation of LAT.

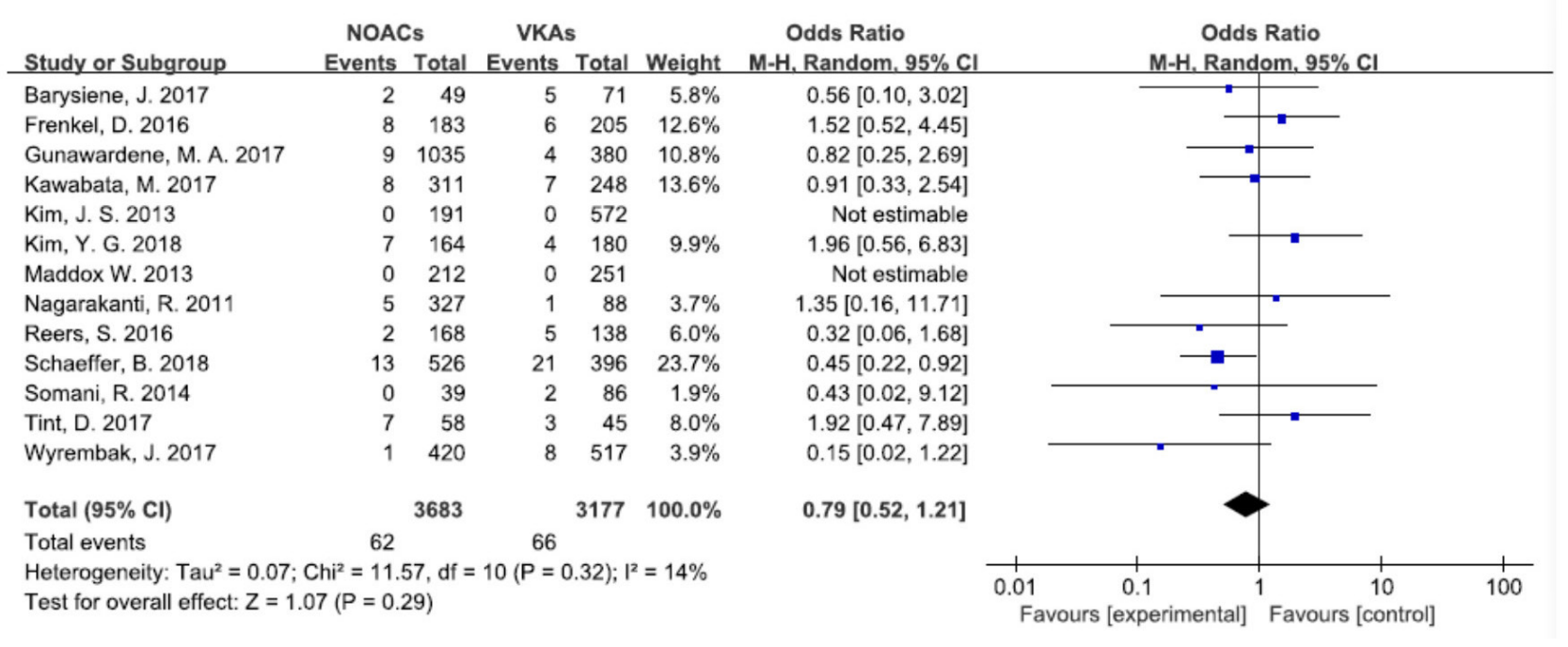

Figure 3. Forest plot for the meta-analysis of the frequencies of atrial thrombus between NOACs (experimental) and VKAs (control). There was no significant difference between the two groups with respect to the formation of LAT.

or VKAs; (3) TEE was used to detect the LAT/LAAT before the cardioversion or catheter ablation; (4) studies comparing NOACs with VKAs in patients with LAT; and (5) randomized controlled and observational study design. The exclusion criteria were the following: (1) studies without VKAs or NOACs as comparator; (2) review, case reports, and letters were excluded; and (3) LAA Occlusion/Excision.

Data extraction and quality assessment: Relevant information from included studies, according to the predefined inclusion criteria, was extracted. Two reviewers independently assessed the quality of included studies, according to the Cochrane Handbook and all disagreements between them were resolved through discussion. We assessed the risk of bias using the Cochrane Risk of Bias tool, which addresses six specific domains: sequence generation, allocation concealment, blinding, incomplete outcome data, no selective outcome reporting, and other sources of bias.

Statistical analysis: For meta-analysis, dichotomous variables were analyzed by using the odds ratios (OR) computed using the Mantel Haenszel method (random models). All results were reported with $95 \%$ confidence intervals (CI). I-square $\left(\mathrm{I}^{2}\right)$ test was used to assess the impact of heterogeneity on the results of the meta-analysis, with $\mathrm{I}^{2}$ values of $0 \%$, $25 \%, 50 \%$ and $75 \%$, respectively, representing no, low, moderate, and high heterogeneity. All analyses were carried out using Review Manager (RevMan) Version 5.3. 


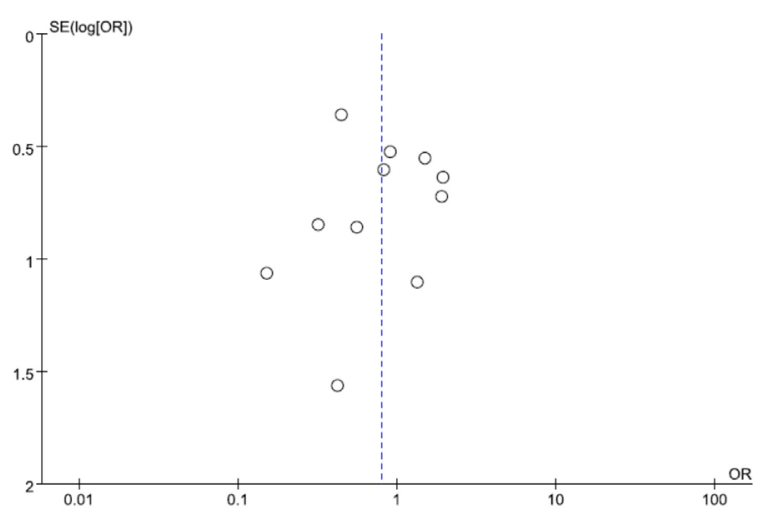

Figure 4. Funnel plots for the publication bias underlying the metaanalyses.

\section{RESULTS}

Included studies: We initially searched 817 records. After removal of duplicates, 693 records were screened. We excluded 594 records after reading the title and abstract. Of the 99 records, 86 records were excluded after the full-text articles were assessed. Thirteen records were assessed for eligibility. Finally, 13 articles that fulfilled the inclusion criteria were identified. The flowchart of the search and selection is illustrated in Figure 1.

Study characteristics and quality evaluation: Overall, 6,860 patients from 13 studies were included in the analysis, of which 3,683 patients were treated with NOACs and 3,177 patients were treated with VKAs [Schaeffer 2018; Reers 2016; Kawabata 2017; Wyrembak 2017; Kim 2013; Maddox 2013; Nagarakanti 2011; Barysienė 2017; Kim 2018; Țînţ 2017; Frenkel 2016; Gunawardene 2017; Somani 2014]. All patients were examined by TEE prior to cardioversion or catheter ablation. The mean age of the patients ranged from $59.9 \pm$ 11.5 to $66.4 \pm 9.79$ years.

The proportion of males ranged from 20 to 83.7. Baseline characteristics of the selected studies are illustrated in Table 1. Among the studies, two studies included the subjects' race characteristics, indicating that there were no significant race differences between warfarin group and the dabigatran group $(P>.05)$ (Table 2). Studies with an $\mathrm{I}^{2}$ statistic of $<25 \%$ was considered to have no heterogeneity. The funnel plot did not indicate a high risk of publication bias (Figure 4).

Meta-analysis: There was no statistically significant difference between the NOACs and VKAs groups with respect to the odds of LAT/LAAT formations (OR 0.79; 95\% CI: $0.52-1.21 ; P=.29 ;\left(I^{2}=14 \%\right)$. Figure 3 shows forest plots presenting included studies. A total of 128 LAT events were reported in the entire study population (62 in NOACs versus 66 in VKAs patients).

Among the 13 reports included in the meta-analysis, there were randomized controlled investigations or observational studies. Of these, one was a controlled investigation, and 12 were observational studies. In observational
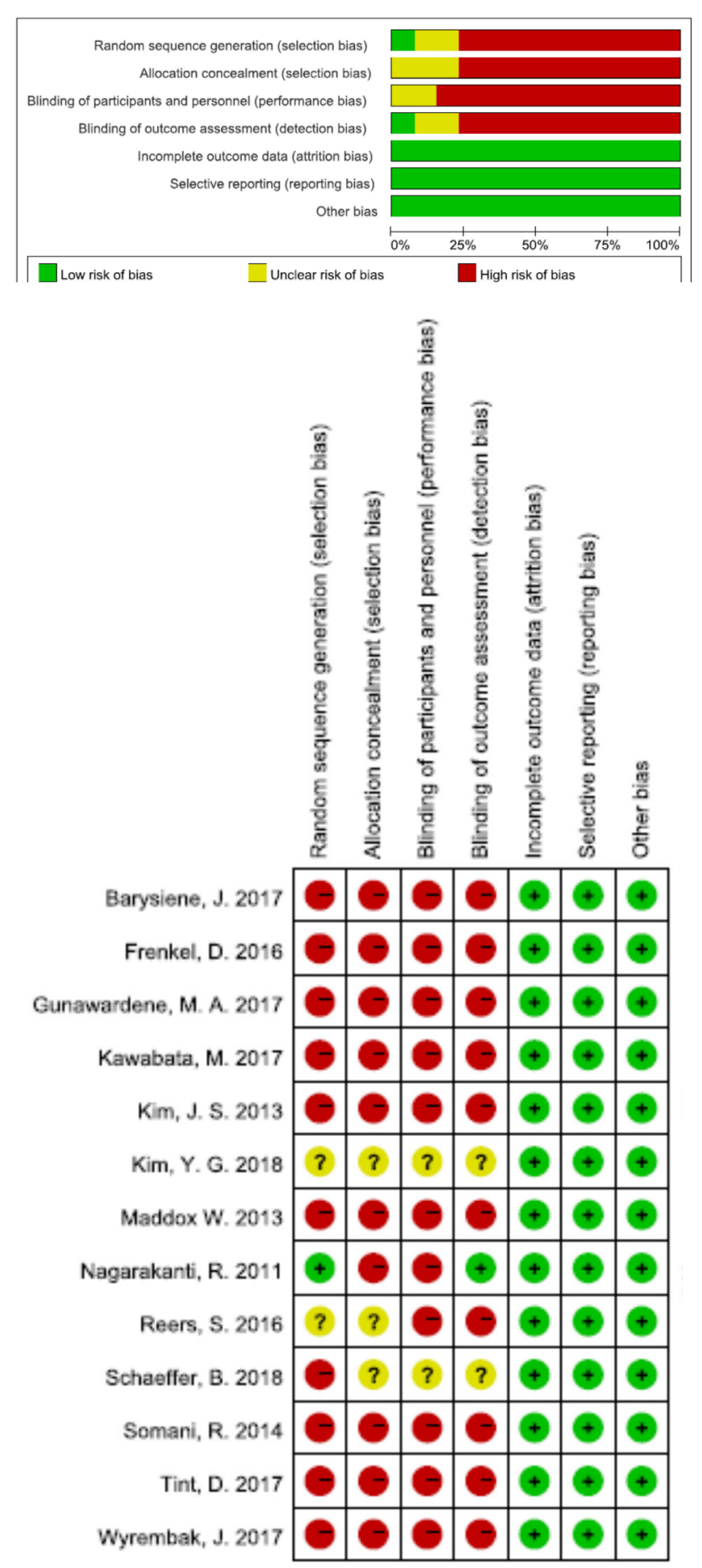

Figure 5. A, Risk of bias graph: review the judgements about each risk of bias item presented as percentages across all included studies. B, Risk of bias summary: review the judgements about each risk of bias item for each included study.

studies, the comparison between NOACs and VKAs revealed no significant difference $(\mathrm{OR}=0.78$; $95 \%$ CI: $0.50-1.23)$ (Figure 2). 
Table 2. Study subjects' race characteristics

\begin{tabular}{|c|c|c|c|c|}
\hline Kim JS, et al 2013 & Number of patients & 191 & 572 & \\
\hline \multirow[t]{2}{*}{ Maddox W, et al 2013} & Number of patients & 212 & 251 & \\
\hline & White race & 195 (92\%) & $233(93 \%)$ & .87 \\
\hline
\end{tabular}

NOACs were as effective as VKAs in the prevention of LAT/LAAT formation in patients with NVAF. Though patients on NOACs therapy showed a lower incidence of LAT/LAAT formation compared with VKAs, it was not significant $(P=.29)$. The $95 \%$ CI covered the one.

\section{DISCUSSION}

The goal of this meta-analysis is to assess the efficacy of NOACs for LAT prevention in non-valvular atrial fibrillation compared with VKAs. The results of our meta-analysis are as follows: The incidence of LAT/LAAT formation in patients undergoing TEE prior to $\mathrm{CV}$ or catheter ablation appeared to be similar in patients with NOACs therapy compared with VKAs therapy (OR 0.79, 95\% CI: $0.52-1.21, P=.29$ ).

Rhythm control is an important strategy for patients with AF, including cardioversion, antiarrhythmic drugs, and radiofrequency catheter [January 2014]. According to guidelines for patients with AF of 48 hours' duration or longer, anticoagulation with warfarin, NOACs (a factor Xa inhibitor or direct thrombin inhibitor) is recommended for at least three weeks before cardioversion [January 2019]. Catheter ablation also is a reasonable rhythm-control strategy for patients with AF. It should be noted that, in the setting of appropriate anticoagulation, the incidence of thrombus formation in $\mathrm{AF}$ patients undergoing TEE prior to catheter ablation or cardioversion should not be ignored. LAT is reported in patients receiving anticoagulation and the screening of LAT in patients with $\mathrm{AF}$ before cardioversion or ablation should be made [Di Minno 2016].

TEE currently is considered the gold standard modality for detecting LAT/LAAT, with high sensitivity and high specificity of 95\%-100\% [Patti 2017; Hahn 2013; Manning 1995], which can be performed before cardioversion to identify the LAT/LAAT [Klein 2001]. However, there still is little solid evidence on the efficacy of NOACs in LAT/LAAT resolution. We conducted meta-analysis to assess the efficacy of NOACs for LAT/LAAT prevention in non-valvular atrial fibrillation compared with VKAs. There are different opinions about the efficacy of prevention of left atrial thrombus between NOACs and VKAs [Yang 2019; Reers 2016]. We reviewed the published metaanalyses. One study indicated similar incidence of thrombus formation in patients with AF between NOACs and VKAs [Reers 2018]. This research included four trials:
ARISTOTLE, ENSURE-AF, RE-LY Dabigatran, and $\mathrm{X}$-VeRT. Of these trials, ENSURE-AF and X-VeRT are prospective randomized clinical trials for electrical cardioversion in AF patients. The results of ENSURE-AF were independent of the TEE-guided strategy [Goette 2016]. Some studies also included the AF patients undergoing cardioversion [Telles-Garcia 2018]. Another study revealed the LAT resolution rate was 63.7\% [Wang 2018]. One study indicated that among initiators of oral anticoagulation, direct-acting oral anticoagulants use was low [Essien 2020]. In this meta-analysis, we included studies that evaluated the efficacy of LAT/LAAT prevention between NOACs and VKAs in patients undergoing cardioversion [Schaeffer 2018; Barysienė 2017; Kim 2018; Ţînţ 2017], or catheter ablation [Frenkel 2016; Gunawardene 2017; Somani 2014], or both [Reers 2016]. Additionally, some studies focus on the AF patients undergoing cardiac surgery, elective cardioversion, or catheter ablation.

GDF-15 is associated with AF-related complications [Zhou 2019]. This meta-analysis provided insights into the effects of the anticoagulation on incidence of thrombus in AF patients.

In one study, comparing with White, African-American race is one of predictors of persistence on warfarin [Jackson 2018]. Another previous trial reviewed data from 30 trials of NOACs, revealing that 21 trials reported race/ethnicity data [Jackson 2015]. It was notable that approximately 2\% were Black in enrollment by race [Jackson 2015]. The relative efficacy of NOACs in minority populations is unknown [Jackson 2015]. In the present analysis, we tried to provide a review of the two studies, including the subjects' race characteristics. We found that there were no significant race differences between the warfarin group and dabigatran group at baseline.

Our study has some limitations. Most of the studies included had small sample sizes. The studies also had different protocols. Furthermore, most data came from observational studies. The analysis of the pooled data showed a similar effect of NOACs, in terms of incidence of LAT/LAAT formations compared with the VKAs. Though patients on NOACs therapy showed a lower incidence of thrombus compared with VKAs, it was not significant. Whether the potential benefit could be proved in future, well-designed studies is not known. Moreover, whether the LAT/LAAT have been in existence prior to anticoagulant therapy is not clear because the TEE was not necessary before the initial anticoagulant therapy. 


\section{CONCLUSION}

Taken together, these results suggest the incidence of thrombus was similar for AF patients on NOACs and VKAs.

\section{REFERENCES}

Barysienė J, Žebrauskaitè A, Petrikonytè D, Marinskis G, Aidietienė S, Aidietis A. 2017. Findings of transoesophageal echocardiogram in appropriately anticoagulated patients with persistent atrial fibrillation prior to planned cardioversion. BMC Cardiovasc Disord. 17(1):67.

Bertaglia E, Anselmino M, Zorzi A, et al. 2017. NOACs and atrial fibrillation: Incidence and predictors of left atrial thrombus in the real world. Int J Cardiol. 249:179-183.

Di Minno MN, Ambrosino P, Dello Russo A, Casella M, Tremoli E, Tondo C. 2016. Prevalence of left atrial thrombus in patients with nonvalvular atrial fibrillation. A systematic review and meta-analysis of the literature. Thromb Haemost. 115(3):663-677.

Essien UR, Magnani JW, Chen N, Gellad WF, Fine MJ, Hernandez I. 2020. Race/Ethnicity and Sex-Related Differences in Direct Oral Anticoagulant Initiation in Newly Diagnosed Atrial Fibrillation: A Retrospective Study of Medicare Data. J Natl Med Assoc. 112(1):103-108.

Frenkel D, D'Amato SA, Al-Kazaz M, et al. 2016. Prevalence of Left Atrial Thrombus Detection by Transesophageal Echocardiography: A Comparison of Continuous Non-Vitamin K Antagonist Oral Anticoagulant Versus Warfarin Therapy in Patients Undergoing Catheter Ablation for Atrial Fibrillation. JACC Clin Electrophysiol. 2(3):295-303.

Goette A, Merino JL, Ezekowitz MD, et al. 2016. Edoxaban versus enoxaparin-warfarin in patients undergoing cardioversion of atrial fibrillation (ENSURE-AF): a randomised, open-label, phase 3b trial [published correction appears in Lancet. 2016 Oct 22;388(10055):1984]. Lancet. 388(10055):1995-2003.

Gunawardene MA, Dickow J, Schaeffer BN, et al. 2017. Risk stratification of patients with left atrial appendage thrombus prior to catheter ablation of atrial fibrillation: An approach towards an individualized use of transesophageal echocardiography. J Cardiovasc Electrophysiol. 28(10):1127-1136.

Hahn RT, Abraham T, Adams MS, et al. 2013. Guidelines for performing a comprehensive transesophageal echocardiographic examination: recommendations from the American Society of Echocardiography and the Society of Cardiovascular Anesthesiologists. J Am Soc Echocardiogr. 26(9):921-964.

Higgins JPT, Green S, editors. 2011. Cochrane handbook for systematic reviews of interventions. New York: Wiley.

Jackson LR 2nd, Kim S, Shrader P, et al. 2018. Early therapeutic persistence on dabigatran versus warfarin therapy in patients with atrial fibrillation: results from the Outcomes Registry for Better Informed Treatment of Atrial Fibrillation (ORBIT-AF) registry. J Thromb Thrombolysis. 46(4):435-439.

Jackson LR 2nd, Peterson ED, Okeagu E, Thomas K. 2015. Review of race/ethnicity in non-vitamin $\mathrm{K}$ antagonist oral anticoagulants clinical trials. J Thromb Thrombolysis. 39(2):222-227.

January CT, Wann LS, Alpert JS, et al. 2014. 2014 AHA/ACC/HRS guideline for the management of patients with atrial fibrillation: a report of the American College of Cardiology/American Heart Association Task Force on practice guidelines and the Heart Rhythm Society [published correction appears in Circulation. 2014 Dec 2;130(23):e272-4].
Circulation. 130(23):e199-e267.

January CT, Wann LS, Calkins H, et al. 2019. 2019 AHA/ACC/HRS Focused Update of the 2014 AHA/ACC/HRS Guideline for the Management of Patients With Atrial Fibrillation: A Report of the American College of Cardiology/American Heart Association Task Force on Clinical Practice Guidelines and the Heart Rhythm Society in Collaboration With the Society of Thoracic Surgeons [published correction appears in Circulation. 2019 Aug 6;140(6):e285]. Circulation. 140(2):e125-e151.

Kawabata M, Goya M, Sasaki T, et al. 2017. Left Atrial Appendage Thrombi Formation in Japanese Non-Valvular Atrial Fibrillation Patients During Anticoagulation Therapy - Warfarin vs. Direct Oral Anticoagulants. Circ J. 81(5):645-651.

Kim JS, She F, Jongnarangsin K, et al. 2013. Dabigatran vs warfarin for radiofrequency catheter ablation of atrial fibrillation. Heart Rhythm. 10(4):483-489.

Kim YG, Choi JI, Kim MN, et al. 2018. Non-vitamin K antagonist oral anticoagulants versus warfarin for the prevention of spontaneous echocontrast and thrombus in patients with atrial fibrillation or flutter undergoing cardioversion: A transesophageal echocardiography study. PLoS One. 13(1):e0191648

Klein AL, Grimm RA, Murray RD, et al. 2001. Use of transesophageal echocardiography to guide cardioversion in patients with atrial fibrillation. N Engl J Med. 344(19):1411-1420.

Maddox W, Kay GN, Yamada T, et al. 2013. Dabigatran versus warfarin therapy for uninterrupted oral anticoagulation during atrial fibrillation ablation. J Cardiovasc Electrophysiol. 24(8):861-865.

Manning WJ, Weintraub RM, Waksmonski CA, et al. 1995. Accuracy of transesophageal echocardiography for identifying left atrial thrombi. A prospective, intraoperative study. Ann Intern Med. 123(11):817-822.

Moher D, Liberati A, Tetzlaff J, Altman DG; PRISMA Group. 2009. Preferred reporting items for systematic reviews and meta-analyses: the PRISMA statement. Ann Intern Med. 151(4):264-W64.

Nagarakanti R, Ezekowitz MD, Oldgren J, et al. 2011. Dabigatran versus warfarin in patients with atrial fibrillation: an analysis of patients undergoing cardioversion. Circulation. 123(2):131-136.

Patti G, Pengo V, Marcucci R, et al. 2017. The left atrial appendage: from embryology to prevention of thromboembolism. Eur Heart J. 38(12):877-887.

Reers S, Agdirlioglu T, Kellner M, et al. 2016. Incidence of left atrial abnormalities under treatment with dabigatran, rivaroxaban, and vitamin K antagonists. Eur J Med Res. 21(1):41.

Reers S, Karanatsios G, Borowski M, Kellner M, Reppel M, Waltenberger J. 2018. Frequency of atrial thrombus formation in patients with atrial fibrillation under treatment with non-vitamin $\mathrm{K}$ oral anticoagulants in comparison to vitamin $\mathrm{K}$ antagonists: a systematic review and metaanalysis. Eur J Med Res. 23(1):49.

Schaeffer B, Rüden L, Salzbrunn T, et al. 2018. Incidence of intracardiac thrombus formation prior to electrical cardioversion in respect to the mode of oral anticoagulation. J Cardiovasc Electrophysiol. 29(4):537-547.

Schotten U, Verheule S, Kirchhof P, Goette A. 2011. Pathophysiological mechanisms of atrial fibrillation: a translational appraisal [published correction appears in Physiol Rev. 2011 Oct;91(4):1533]. Physiol Rev. 91(1):265-325.

Somani R, Mohajer K, Haley C, et al. 2014. The peri-procedural use of dabigatran in patients undergoing left atrial ablation for atrial fibrillation. Cardiovasc Ther. 32(5):198-201. 
Telles-Garcia N, Dahal K, Kocherla C, Lip GYH, Reddy P, Dominic P. 2018. Non-vitamin K antagonists oral anticoagulants are as safe and effective as warfarin for cardioversion of atrial fibrillation: A systematic review and meta-analysis. Int J Cardiol. 268:143-148.

Ţînţ D, Petriş AO, Pop I, Melnic R, Ignat AM, Rogozea LM. 2017. Vitamin K Antagonists Versus Novel Oral Anticoagulants for Elective Electrical Cardioversion of Atrial Fibrillation. Am J Ther. 24(5):e553-e558.

Wallace TW, Atwater BD, Daubert JP, et al. 2010. Prevalence and clinical characteristics associated with left atrial appendage thrombus in fully anticoagulated patients undergoing catheter-directed atrial fibrillation ablation. J Cardiovasc Electrophysiol. 21(8):849-852.

Wang NC, Sather MD, Hussain A, et al. 2018. Oral anticoagulation and left atrial thrombi resolution in nonrheumatic atrial fibrillation or flutter:
A systematic review and meta-analysis. Pacing Clin Electrophysiol. 41(7):767-774.

Wyrembak J, Campbell KB, Steinberg BA, et al. 2017. Incidence and Predictors of Left Atrial Appendage Thrombus in Patients Treated With Nonvitamin K Oral Anticoagulants Versus Warfarin Before Catheter Ablation for Atrial Fibrillation. Am J Cardiol. 119(7):1017-1022.

Yang J, Zhang X, Wang XY, Zhang C, Chen SZ, Hu SJ. 2019. Comparison of transesophageal echocardiography findings after different anticoagulation strategies in patients with atrial fibrillation: a systematic review and meta-analysis. BMC Cardiovasc Disord. 19(1):261.

Zhou J, Kang ZF,Liu LL, et al. 2019. Predicting Value of Growth Differentiation Factor 15 and Its Correlations With Atrial Fibrillation. The Heart Surgery Forum 23: E452-E460. 